Decision Making in Manufacturing and Services

VOL. $1 \bullet 2007 \bullet$ NO. 1-2 • PP. 153-173

\title{
Advances in Cone-Based Preference Modeling for Decision Making with Multiple Criteria
}

\author{
Margaret M. Wiecek*
}

\begin{abstract}
Decision making with multiple criteria requires preferences elicited from the decision maker to determine a solution set. Models of preferences, that follow upon the concept of nondominated solutions introduced by Yu (1974), are presented and compared within a unified framework of cones. Polyhedral and nonpolyhedral, convex and nonconvex, translated, and variable cones are used to model different types of preferences. Common mathematical properties of the preferences are discussed. The impact of using these preferences in decision making is emphasized.
\end{abstract}

Keywords: cones, preferences, nondominated solutions, Pareto solutions, multiple criteria, decision making

Mathematics Subject Classification: 90C29, 91B08.

Received/Revised: 13 June 2007/1 September 2007

\section{INTRODUCTION}

Rapid technological and economic growth over the last fifty years has changed human lives and made modern society face complex decision making problems. In the present world, people have to deal with urbanization and industrialization, increase of water and energy demands, environmental pollution, shortage of natural resources and food, and many other challenges. These problems necessitate the development of multidisciplinary approaches for analyzing diverse mechanisms and consequences of modern civilization.

Multiple criteria decision making (MCDM), as a subfield of systems engineering and science, has become a modeling and methodological tool for dealing with complex decision making problems encountered in many areas of human activity in business, management, and engineering. The development of MCDM models and methods has been motivated not only by a variety of real-life problems requiring the consideration of multiple criteria, but also by the scientists' and engineers' desire to propose enhanced decision making techniques using recent advancements in mathematical optimization, scientific computing, and computer technology.

\footnotetext{
* Department of Mathematical Sciences, Clemson University, Clemson, SC USA. E-mail: wmalgor@clemson.edu
} 
A complex decision making problem is characterized by multiple objectives or criteria such as distance, time, cost, reliability, maintenance, safety, productivity, performance, affordability, and many others. In the presence of multiple criteria, a unique optimal decision for the problem does not exist but rather many or even infinitely many decisions are suitable. MCDM includes two complementary areas: mathematics-based multiple objective programming (MOP) and decision maker-driven multiple criteria decision analysis (MCDA). The goal of MOP is to find suitable solutions of mathematical programs with multiple objective functions. Since in general, objectives are noncomparable and conflicting, solution sets of multiple objective programs usually include a large or infinite number of points which are referred to as efficient (Pareto, noninferior, nondominated) solutions or decisions. Trading one efficient decision for another results in improvement of at least one objective and simultaneous deterioration of at least one other. MOP constitutes the first phase of MCDM and includes generating efficient decisions as well as characterizing the efficient (nondominated) set.

The next phase of the decision making process, MCDA, encompasses decision makers' judgments and preferences to derive a preferred decision from among the efficient (nondominated) solutions that will become the policy to be implemented for the problem.

In this paper we focus on the stage of MOP and investigate the concept of optimality introduced by $\mathrm{Yu}$ (1974). In his seminal paper, Yu proposes to use convex cones to model decision maker's (DM's) preferences and defines domination cones and nondominated solutions to determine solution sets for multiple objective programs.

Some researchers undertake efforts to generalize the convex-cone approach of $\mathrm{Yu}$ into sets and other objects. Bergstresser et al. (1976) use convex sets rather than convex cones to represent preferences. Lin (1976) provides a comparison of the defined optimality concepts and Chew (1979) proposes a reformulation for general vector spaces. Takeda and Nishida (1980) introduce fuzzy domination structures for MOP while Hazen and Morin (1983) study optimality conditions for MOP with a nonconical order. Many of these earlier results are collected in the monograph by $\mathrm{Yu}$ (1985). Later, Weidner $(2001,2003)$ studies scalarization approaches to multiobjective programs with preferences modeled by parameter-depending sets, Chen and Yang (2002) relate a variable domination structure to a nonlinear scalarization for MOP, and Chen et al. (2005) examine variable dominations structures for set-valued optimization problems. Wu (2004) further examines the relevance of convex cones for a solution concept in fuzzy MOP.

The purpose of this paper is to present the state-of-the-art in cone-based preference modeling for decision making with multiple criteria. The models either refine or go beyond the existing framework of convex cones and show how convex analysis can be successfully employed in decision making. The paper is expected to lay out an overall theoretical foundation for decision making with multiple criteria which could be implemented in applied studies in various disciplines including, among others, manufacturing and services.

Section 2 provides some preliminaries that establish the classical concepts of nondominance. In Section 3, we present two models of relative importance which are 
developed with polyhedral cones. These models are applicable to decision making situations in which the DM is willing and able to quantify tradeoffs between the criteria to model their relative importance among each other. In Section 4, we relax nondominance to study approximate nondominance and we accomplish this by using translated cones. In Section 5, we go beyond polyhedral cones and work with cones induced by positively homogenous functions to discover that the earlier discovered properties still hold even that polyhedrality does not. Finally in Section 6, we show that variable cones are needed to model the preference of equitability. We conclude the paper in Section 7.

\section{PRELIMINARIES}

Throughout this article the following notation is used. Let $\mathbb{R}^{m}$ be a Euclidean vector space and $y^{1}, y^{2} \in \mathbb{R}^{m} . y^{1}<y^{2}$ denotes $y_{i}^{1}<y_{i}^{2}$ for all $i=1, \ldots, m . y^{1} \leqq y^{2}$ denotes $y_{i}^{1} \leq y_{i}^{2}$ for all $i=1, \ldots, m$. $y^{1} \leq y^{2}$ denotes $y^{1} \leqq y^{2}$ but $y^{1} \neq y^{2}$.

\subsection{CONES}

A cone $C \subset \mathbb{R}^{m}$ is a nonempty set for which $\mathbf{d} \in C \Rightarrow \lambda \mathbf{d} \in C$ whenever $\lambda>0$. It is said to be convex if $\mathbf{d}^{1}, \mathbf{d}^{2} \in C \Rightarrow \mathbf{d}^{1}+\mathbf{d}^{2} \in C$, and pointed if $\sum_{i=1}^{k} \mathbf{d}^{i}=0 \Rightarrow \mathbf{d}^{i}=0$ for all $i=1, \ldots, k$, where the $\mathbf{d}^{i} \in C$ are any $k$ elements of $C$. Let $C^{o}:=C \backslash\{\mathbf{0}\}$.

Definition 2.1. Let $A \in \mathbb{R}^{l \times m}$ be a matrix. The polyhedral cone $C(A) \subset \mathbb{R}^{m}$ determined by $A$ is defined by

$$
C(A):=\left\{\mathbf{d} \in \mathbb{R}^{m}: A \mathbf{d} \geqq \mathbf{0}\right\} .
$$

This representation of the polyhedral cone as the solution set of a homogeneous system of linear inequalities with the $l \times m$ coefficient matrix $A$ is called the inequality form of the cone. In particular, for $A=I^{m} \in \mathbb{R}^{m \times m}$ the $m$-dimensional identity matrix, the polyhedral cone $C\left(I^{m}\right):=\left\{\mathbf{d} \in \mathbb{R}^{m}: \mathbf{d} \geqq \mathbf{0}\right\}=: \mathbb{R}_{\geqq}^{m}$.

Proposition 2.1. Let $A \in \mathbb{R}^{l \times m}$ be a matrix and $C(A) \subset \mathbb{R}^{m}$ be the related polyhedral cone. $C(A)$ is always convex. $C(A)$ is pointed if and only if the mapping $\mathbf{d} \mapsto A \mathbf{d}$ is injective.

The equivalent conditions for the pointedness of a polyhedral cone $C(A) \subset \mathbb{R}^{m}$ are that $\operatorname{rank} A=m \geq 2$ or the linear mapping $\mathbf{d} \mapsto A \mathbf{d}$ is injective (i.e., $A \mathbf{d}=0$ if and only if $\mathbf{d}=\mathbf{0}$ ). In particular, the cone $C\left(I^{m}\right)=\mathbb{R}_{\geqq}^{m}$ is convex and pointed.

\subsection{NONDOMINATED OUTCOMES}

The MOP framework includes the following basic elements: a solution space, an outcome space, a collection of objective (criterion) functions (performance indices) evaluating solutions and producing outcomes. The goal is to identify those feasible solutions that yield the most satisfactory (preferred) outcome(s) according to DM's 
preferences. In this study we assume that the spaces are Euclidean, the objective functions are real-valued, and the preferences are modeled with cones.

More specifically, let $\mathbb{R}^{n}$ and $\mathbb{R}^{m}$ be the solution (decision, design) space and the objective (criterion, outcome, performance) space, respectively. Let $X \subseteq \mathbb{R}^{n}$ be a set of feasible solutions in $\mathbb{R}^{n}$. Let the vector-valued function $f: X \rightarrow \mathbb{R}^{m}$ be composed of $m$ real-valued functions $f_{i}: X \rightarrow \mathbb{R}, i=1, \ldots, m$. The set $Y \subset \mathbb{R}^{m}$ of accessible outcomes is defined as $Y:=f(X)=\left\{\mathbf{y} \in \mathbb{R}^{m}: y_{i}=f_{i}(\mathbf{x}), i=1, \ldots, m, x \in X\right\}$. It is of interest to find outcomes that perform satisfactorily according to DM's preferences modeled with an ordering cone. In other words, we intend to optimize all criteria while the "optimality" in the $m$-dimensional objective space is determined by a cone.

Let $C \subset \mathbb{R}^{m}$ be an ordering cone in the outcome space. We assume that it is the set of all dominated directions in $\mathbb{R}^{m}$ and refer to it as the domination cone $D$. The notion of domination cone was introduced into MOP by $\mathrm{Yu}$ (1974). A domination cone contains all vectors $\mathbf{d} \in \mathbb{R}^{m}$ such that for $\mathbf{y}, \mathbf{y}^{1} \in Y$, if $\mathbf{y}^{1}=\mathbf{y}+\mathbf{d}$ for some $\mathbf{d} \in D^{\circ}$, then $\mathbf{y}^{1}$ is dominated by $\mathbf{y}$. The vectors in the domination cone can be thought of as "bad" or "dominated" directions to travel within $\mathbb{R}^{m}$. A nondominated outcome is one that is not dominated by any other outcome in $Y$.

Definition 2.2. An element $\mathbf{y} \in Y$ is called a nondominated element of the set $Y$ with respect to the domination cone $D$ if there do not exist an element $\mathbf{y}^{1} \in Y$ and a direction $\mathbf{d} \in D^{\circ}$ such that $\mathbf{y}=\mathbf{y}^{1}+\mathbf{d}$, or equivalently, $Y \cap\left(\mathbf{y}-D^{\circ}\right)=\emptyset$. The set of all nondominated elements of $Y$ with respect to $D$ is denoted by $N(Y, D)$. The set of weakly nondominated elements of $Y$ with respect to $D$ is defined as $N_{w}(Y, D):=$ $N(Y$, int $D)$.

The cone $C$ can also be defined as the set of all preferred directions in $\mathbb{R}^{m}$ and then it is referred to as the preference cone $P$. The cone $P$ is then the set of all directions $\mathbf{d} \in \mathbb{R}^{m}$ such that for $\mathbf{y}, \mathbf{y}^{1} \in Y$, if $\mathbf{y}^{1}=\mathbf{y}+\mathbf{d}$ for some $\mathbf{d} \in P^{\circ}$, then $\mathbf{y}$ is dominated by $\mathbf{y}^{1}$. In other words, a preference cone contains all "good" or "preferred" directions to travel within $\mathbb{R}^{m}$.

Definition 2.3. An element $\mathbf{y} \in Y$ is called a nondominated element of the set $Y$ with respect to the preference cone $P$ if there do not exist an element $\mathbf{y}^{1} \in Y$ and a direction $\mathbf{d} \in P^{\circ}$ such that $\mathbf{y}=\mathbf{y}^{1}-\mathbf{d}$, or equivalently, $Y \cap\left(\mathbf{y}+P^{\circ}\right)=\emptyset$. The set of all nondominated elements of $Y$ with respect to $P$ is denoted by $N(Y, P)$. The set of weakly nondominated elements of $Y$ with respect to $P$ is defined as $N_{w}(Y, P):=$ $N(Y$, int $P)$.

The relationship between the domination and preference cones is given as $D=-P$ since the negation of a dominated direction in $D$ must be a preferred direction in $P$ to maintain consistency of preferences. Typically, it is assumed that the cones are convex and pointed. In other words, the sum of two dominated (preferred) directions $\mathbf{d}^{1}, \mathbf{d}^{2} \in D(P)$ is again a dominated (preferred) direction, $\mathbf{d}^{1}+\mathbf{d}^{2} \in D(P)$ and that if both $\mathbf{d}$ and $-\mathbf{d} \in D(P)$ are dominated (preferred) directions, then $\mathbf{d}=\mathbf{0}$. We will see later that these assumptions may not be fulfilled for a certain class of preferences.

In general, one can approach preference modeling using domination cones or preference cones and seek the sets $N(Y, D)$ or $N(Y, P)$. Some authors (e.g., Hunt (2004)) 
use the latter since they believe that it is more intuitive for DMs to express what they like or prefer as oppose to what they do not like or do not prefer.

Alternative terminology refers to the nondominated sets as sets of minimal or maximal elements defined for partially ordered sets. However, we choose to follow the terminology of $\mathrm{Yu}(1974)$ since we believe it has been very effective theoretically and is intuitive for DMs, and therefore more attractive.

\subsection{NONDOMINANCE WITH RESPECT TO POLYHEDRAL CONES}

As indicated above, the simplest polyhedral cone is generated by the $m \times m$ identity matrix which yields the cone being the nonnegative orthant in $\mathbb{R}^{m}$. In the literature, this cone is known as the Pareto cone. The Pareto domination cone $D_{P a r} \subset \mathbb{R}^{m}$ is defined by

$$
D_{\text {Par }}:=\mathbb{R}_{\geqq}^{m}
$$

and the Pareto preference cone $P_{P a r} \subset \mathbb{R}^{m}$ is given as

$$
P_{\text {Par }}=-D_{\text {Par }}
$$

An overwhelming majority of studies in MOP and MCDM use the notion of Pareto nondominance based on the Pareto domination and preference cones. It is however very straightforward to generalize the Pareto cone to polyhedral cones.

Definition 2.4. Let $A \in \mathbb{R}^{l \times m}$ be a matrix. The domination cone $D(A) \subset \mathbb{R}^{m}$ determined by $A$ is defined by

$$
D(A):=\left\{\mathbf{d} \in \mathbb{R}^{m}: A \mathbf{d} \geqq \mathbf{0}\right\} .
$$

The preference cone $P(A) \subset \mathbb{R}^{m}$ is given as

$$
P(A)=-D(A)
$$

The following result is well established throughout the literature, see (Sawaragi, et. al, 1985; Yu, 1985; Noghin, 1997; Cambini et. al, 2003; Hunt, Wiecek, 2003) among others.

Theorem 2.1. Let $A \in \mathbb{R}^{l \times m}$ be a matrix and $D(A) \subset \mathbb{R}^{m}$ be the related domination cone. Then

$$
A\left[\mathrm{~N}(Y, D(A)] \subseteq \mathrm{N}\left(A[Y], \mathbb{R}_{\geqq}^{l}\right)\right.
$$

where $A[Y]=\{\mathbf{z}: \mathbf{z}=A \mathbf{y}, \mathbf{y} \in Y\}$. Furthermore, if the cone $D(A)$ is pointed, then

$$
A\left[\mathrm{~N}(Y, D(A)]=\mathrm{N}\left(A[Y], \mathbb{R}_{\geqq}^{l}\right)\right.
$$

In any case,

$$
A\left[\mathrm{~N}\left(Y_{w}, D(A)\right]=\mathrm{N}_{w}\left(A[Y], \mathbb{R}_{\geqq}^{l}\right)\right.
$$

Hence, the problem of finding the nondominated set of $Y$ with respect to a domination cone $D(A)$ is equivalent to finding the nondominated set of $A[Y]$ with respect to the Pareto cone, where $A$ is the matrix that determines the domination cone. 


\section{RELATIVE IMPORTANCE OF CRITERIA}

In the following section we investigate how the structure of a matrix $A$ determining the polyhedral cone may influence the set of nondominated solutions with respect to the related domination cone. In particular, we use the elements of this matrix to model relative importance of the $m$ criteria that evaluate the set of feasible solutions.

Even that one seeks to simultaneously optimize all criteria over the feasible set, some of them may be considered more important than others. Using the lexicographic ordering to model relative importance of criteria has been perhaps the first approach undertaken in the literature. Doležal (1976) applies the lexicographic ordering to biobjective programs in which one criterion is more important than the other, while Ying (1983) applies the lexicographic ordering to multiobjective programs whose criteria are divided into groups of equal importance. A large number of researchers have modeled relative importance of criteria with numerical weights assigned to the criteria to express their importance. Podinovskii $(1977,1978,1994,2000)$ defines preference and indifference relations using coefficients of relative importance. Similar concepts are used by Menshikova and Podinovskii (1988) and Roy and Mousseau (1996). Berman and Naumov (1989) introduce interval tradeoffs between criteria which are defined as the quantity by which one criterion must be improved to compensate for the decay in another criterion. They use the tradeoffs to construct a polyhedral cone modeling DM's preferences among criteria. Noghin (1997) and Noghin and Tolstykh (2000) divide criteria into two groups, more and less important, and construct weight functions to define coefficients of this relative importance. Their approach leads to the augmentation of the Pareto cone to a polyhedral cone subsuming it. Wei et al. (2000) discuss relative importance of criteria from the perspective of group decision-making while Karaskal and Michalowski (2003) recognize that the importance may change during the decision-making process and may depend on current values of criteria.

In our work, in order to model DM's preferences, we partially follow upon Noghin (1997), since we put all criteria into two groups, and upon Berman and Naumov (1989) as we explicitly use the matrix description of a polyhedral cone. We assume that the DM follows the Pareto preference in the outcome space $\mathbb{R}^{m}$ implying that every direction in the preference Pareto cone is a preferred direction and thus is always contained in the DM's overall preference cone. Furthermore, when traveling along a direction $\mathbf{d} \in \mathbb{R}^{m}$ neither in the Pareto preference cone nor in the Pareto domination cone, the DM recognizes simultaneous increase and decrease of values of particular components $d_{i}, i=1, \ldots, m$, which the DM refers to as decay and improvement in this component, respectively.

If the DM is willing to allow tradeoffs between criteria, then additional attractive directions may be appended to the Pareto cone to construct the DM's overall preference cone.

Definition 3.1. An allowable tradeoff between criteria $i$ and $j, i, j \in\{1, \ldots, m\}$, $i \neq j$, denoted $a_{i j}$, is the largest amount of decay in criterion $i$ considered allowable to the DM to gain one unit of improvement in criterion $j$. Also, $a_{i j} \geq 0$ for all $i$ and $j, i \neq j$. 
The values of the allowable tradeoffs depend on DM's preferences. We assume that an experienced DM has previous knowledge of and experience with the decision problem to guide the assignment of allowable tradeoff values. In particular, if $a_{i j}=0$ for all $i, j \in\{1, \ldots, m\}, i \neq j$, then the DM has the classical Pareto preference.

A tradeoff between two criteria incurred when traveling along a direction in the outcome space is called a directional tradeoff.

Definition 3.2. A directional tradeoff between criteria $i$ and $j, i, j \in\{1, \ldots, m\}$, $i \neq j$, denoted $t_{i j}(\mathbf{d})$, is the tradeoff incurred between criteria $i$ and $j$ when traveling along direction $\mathbf{d} \in \mathbb{R}^{m}$ and is defined as follows:

$$
\begin{aligned}
& t_{i j}(\mathbf{d})=0 \quad \text { if } d_{i} \leq 0 \text { and } d_{j} \leq 0 \\
& t_{i j}(\mathbf{d})=\frac{d_{i}}{-d_{j}} \quad \text { if } d_{i}>0 \text { and } d_{j}<0 \\
& t_{i j}(\mathbf{d})=\infty \quad \text { if } d_{i} \geq 0 \text { and } d_{j} \geq 0, \mathbf{d} \neq \mathbf{0} \\
& t_{i j}(\mathbf{d}) \quad \text { is undefined otherwise }
\end{aligned}
$$

Given the definition of an allowable tradeoff and a directional tradeoff between two criteria, we define attractive directions in the outcome space.

Definition 3.3. A direction $\mathbf{d} \in \mathbb{R}^{m}$ is an attractive direction in the outcome space if $t_{i j}(\mathbf{d}) \leq a_{i j}$ for every pair of criteria $i$ and $j, i, j \in\{1, \ldots, m\}, i \neq j$.

In other words, a direction $\mathbf{d} \in \mathbb{R}^{m}$ is an attractive direction if every directional tradeoff between criteria $i$ and $j$ with respect to $\mathbf{d}$ is no larger than the corresponding allowable tradeoff between criteria $i$ and $j$.

In the following subsections we present two types of matrix $A$ to model relative importance of criteria. The models require eliciting preferences from the DM in the form of allowable tradeoffs between criteria.

\subsection{MODEL 1}

In the first model, we assume that the DM allows one criterion $i \in\{1,2, \ldots, m\}$ to decay only if all other criteria $j \in\{1,2, \ldots, m\}, j \neq i$, improve. The DM is required to define an allowable tradeoff $a_{i j}$ for every $j \in\{1,2, \ldots, m\}, j \neq i$. It may be of interest in some cases to repeat this process with more than one selection of criterion $i$, thus the model is constructed to address this possibility. All attractive directions in $\mathbb{R}^{m}$ are appended to the Pareto cone to obtain the DM's new preference cone.

Let $l \in\{1,2, \ldots, m\}$ represent the criterion that the DM would like to decay while all other criteria $j \in\{1,2, \ldots, m\}, j \neq l$, improve, and let the tradeoff between the improvement and each decay be bounded by some allowable tradeoff $a_{l j}$. Define the set of all attractive directions to the DM

$P_{l}:=\left\{\mathbf{d} \in \mathbb{R}^{m} \mid d_{l}>0, d_{j}<0\right.$ for all $j \in\{1,2, \ldots, m\}, j \neq l$, and $t_{l j}(\mathbf{d}) \leq a_{l j}$ for all $\left.j \neq l\right\}$

Then the preference cone of Model 1 is then defined

$$
P_{1}:=\bigcup_{l} P_{l} \cup\left(-\mathbb{R}_{\geqq}^{m}\right)
$$


and the domination cone of Model 1 is given by

$$
D_{1}=-P_{1}
$$

Note that if $\mathbf{d} \in P_{l}$ for some $l \in\{1,2, \ldots, m\}$ such that $d_{l}>0$ and $-\infty<d_{j}<$ $0, j \in\{1,2, \ldots, m\}, j \neq l$, then $a_{l j}>0$ for all $j \in\{1,2, \ldots, m\}, j \neq l$.

We now present the algebraic cone representations of $D_{1}$. After all allowable tradeoff values are collected from the DM, the following matrix is constructed.

Definition 3.4. Let $A_{1}$ be an $m(m-1) \times m$ matrix described by $m$ blocks of $m-1$ rows and $m$ columns, where $A_{1}^{i j}$ represents row $j \in\{1,2, \ldots, m-1\}$ of block $i \in\{1,2, \ldots, m\}$ of $A_{1}$, and $\left(A_{1}^{i j}\right)_{k}$ represents the element of $A_{1}^{i j}$ in column $k \in\{1,2, \ldots, m\}$. The elements of $A_{1}$ are defined as follows:

$$
\begin{aligned}
\left(A_{1}^{i j}\right)_{i} & =1 & & \text { for all } i \in\{1,2, \ldots, m\}, j \in\{1,2, \ldots, m-1\} \\
\left(A_{1}^{i j}\right)_{j} & =a_{i j} & & \text { if } j<i, i \in\{1,2, \ldots, m\}, j \in\{1,2, \ldots, m-1\} \\
\left(A_{1}^{i j}\right)_{j+1} & =a_{i(j+1)} & & \text { if } j \geq i, i \in\{1,2, \ldots, m\}, j \in\{1,2, \ldots, m-1\} \\
\left(A_{1}^{i j}\right)_{k} & =0 & & \text { otherwise, } i \in\{1,2, \ldots, m\}, j \in\{1,2, \ldots, m-1\}
\end{aligned}
$$

In general, $A_{1}$ has the following structure:

$$
A_{1}=\left[\begin{array}{ccccc}
1 & a_{12} & 0 & \cdots & 0 \\
1 & 0 & a_{13} & \ddots & \vdots \\
\vdots & \vdots & \ddots & \ddots & 0 \\
1 & 0 & \cdots & 0 & a_{1 m} \\
a_{21} & 1 & 0 & \cdots & 0 \\
0 & 1 & a_{23} & \ddots & \vdots \\
\vdots & \vdots & 0 & \ddots & 0 \\
0 & 1 & 0 & 0 & a_{2 m} \\
\vdots & \vdots & \vdots & \vdots & \vdots \\
a_{m 1} & 0 & \cdots & 0 & 1 \\
0 & a_{m 2} & \ddots & \vdots & 1 \\
\vdots & \ddots & \ddots & 0 & \vdots \\
0 & \cdots & 0 & a_{m(m-1)} & 1
\end{array}\right]_{m(m-1) \times m}
$$


For a more detailed view, the $i$ th block of $A_{1}$ has the following structure:

$$
A_{1}^{i}=\left[\begin{array}{cccccccc}
\multicolumn{7}{c}{\text { column }} \\
a_{i 1} & 0 & \cdots & 0 & 1 & 0 & \cdots & 0 \\
0 & a_{i 2} & \ddots & \vdots & 1 & \vdots & & \vdots \\
\vdots & \ddots & \ddots & 0 & \vdots & \vdots & & \vdots \\
\vdots & & \ddots & a_{i(i-1)} & 1 & 0 & & \vdots \\
\vdots & & & 0 & 1 & a_{i(i+1)} & \ddots & \vdots \\
\vdots & & & \vdots & \vdots & 0 & \ddots & 0 \\
0 & \ldots & \ldots & 0 & 1 & 0 & 0 & a_{i m}
\end{array}\right]_{(m-1) \times m}
$$

Let $C\left(A_{1}\right)$ be a cone represented in inequality representation with matrix $A_{1}$.

$$
C\left(A_{1}\right):=\left\{\mathbf{d} \in \mathbb{R}^{m} \mid A_{1} \mathbf{d} \geqq \mathbf{0}\right\}
$$

Since all entries of $A_{1}$ are nonnegative, $A_{1} \mathbf{d} \geqq \mathbf{0}$ for any $\mathbf{d} \in \mathbb{R}_{\geq}^{m}$ and thus $\mathbb{R}_{\geq}^{m} \subseteq$ $C\left(A_{1}\right)$. Matrix $A_{1}$ consists of $m$ blocks since each criterion $j \in\{1,2, \ldots, m\}$ may be selected to decay. In turn, each block has $m-1$ rows since $m-1$ criteria improve while one criterion decays. If $a_{i j}>0$ for some $i \in\{1,2, \ldots, m\}$ and for all $j \in$ $\{1,2, \ldots, m\}, j \neq i$, then the $i$ th block of $A_{1}$ describes directions $\mathbf{d} \in P_{i}$. If $a_{i j}=0$ for some $i \in\{1,2, \ldots, m\}$ and for any $j \in\{1,2, \ldots, m\}, j \neq i$, then $P_{i}=\emptyset$.

Theorem 3.1. Let $D_{1}$ and $C\left(A_{1}\right)$ be defined as in (2) and (3). If $a_{i j} a_{j i} \leq 1$ for all $i, j \in\{1,2, \ldots, m\}, i \neq j$, then $D_{1}=C\left(A_{1}\right)$.

Since we require preference cones to be convex and pointed to maintain preference consistency, Corollaries 3.1 and 3.2 reveal conditions to ensure $C\left(A_{1}\right)$ possesses these properties.

Corollary 3.1. If $a_{i j} a_{j i} \leq 1$ for all $i, j \in\{1,2, \ldots, m\}, i \neq j$, then the preference cone $C\left(A_{1}\right) \subset \mathbb{R}^{m}$ is convex.

Corollary 3.2. If $m=2$, then the preference cone $C\left(A_{1}\right) \subset \mathbb{R}^{2}$ is pointed if $a_{12} a_{21}<$ 1. If $m \geq 3$, then $C\left(A_{1}\right) \subset \mathbb{R}^{m}$ is pointed.

For a problem with three criteria, $A_{1}$ has three submatrices each of dimension $2 \times 3$ producing the $6 \times 3$ matrix shown in (4). The first two rows of $A_{1}$ form the first submatrix, the third and fourth rows form the second submatrix and the last two rows form the third submatrix.

$$
A_{1}=\left[\begin{array}{ccc}
1 & a_{12} & 0 \\
1 & 0 & a_{13} \\
a_{21} & 1 & 0 \\
0 & 1 & a_{23} \\
a_{31} & 0 & 1 \\
0 & a_{32} & 1
\end{array}\right]
$$


The first submatrix models the case that criterion $f_{1}$ decays while criteria $f_{2}$ and $f_{3}$ improve. The second submatrix models the case that criterion $f_{2}$ decays while criteria $f_{1}$ and $f_{3}$ improve. Finally, the third submatrix models the case that criterion $f_{3}$ decays while criteria $f_{1}$ and $f_{2}$ improve. One might expect that in practical decison making the occurrence of all these three situations is unrealistic. While this theoretical model allows for all these three cases to take place simultaneously, any submatrix may collapsed to the corresponding identity row if the related case should not be included due to a practical context.

\subsection{MODEL 2}

In Model 1, the DM allows only one criterion to decay while all other criteria must improve. In the second preference model, we assume that the DM allows more than one criterion to decay and all the others are expected to improve or remain unchanged. We call this Model 2, which is less restrictive than Model 1 and perhaps more applicable in practice because multiple criteria are allowed to improve or decay at a time.

Model 2 requires that the indices of all criteria be divided into two groups: the set $M$ of indices corresponding to a relatively more important group of criteria that are not allowed to decay and must improve and the set $L$ of indices corresponding to a relatively less important group of criteria that are allowed to decay. Even though the criteria represented by $L$ are allowed to decay, if they also improve or remain unchanged then we consider ourselves fortunate.

The sets $L$ and $M$ are constructed such that $L \cup M=\{1,2, \ldots, m\}$ and $L \cap M=\emptyset$. Also, letting $|L|=l, 0 \leq l \leq m-1$, implies that $|M|=m-l$. The DM is required to define an allowable tradeoff $a_{i j}$ for every pair $i, j, i \neq j$, such that $i \in L$ and $j \in M$. All attractive directions in $\mathbb{R}^{m}$ (see Definition 3.3) are appended to the Pareto cone to obtain the DM's preference cone $P_{2}$. We assume that directions in the Pareto preference cone are always attractive to the DM and are always contained in the preference cone $P_{2}$ defined as follows.

Given the sets $L$ and $M$, define the set

$$
W:=\left\{\mathbf{d} \in \mathbb{R}^{m} \mid d_{i} \leq 0 \text { for all } i \in M \text { and } d_{k} \geq \sum_{i \in M} a_{k i} d_{i} \text { for each } k \in L\right\}
$$

Then the preference cone of Model 2 is defined as

$$
P_{2}:=W \cup\left(-\mathbb{R}_{\geqq}^{m}\right)
$$

and the domination cone of Model 2 is given by

$$
D_{2}=-P_{2}
$$

The components $d_{k}, k \in L$, of directions $\mathbf{d} \in W$ are allowed to be nonnegative to represent decay or no change in the corresponding criteria. However, these components are also allowed to be negative to represent improvement in these criteria, 
which is an attractive feature. The components $d_{i}, i \in M$, of directions $\mathbf{d} \in W$ are only allowed to be nonpositive because the corresponding criteria are considered relatively more important and are never allowed to decay.

According to the definition of set $W$ given in (5), the total amount of improvement allowed for each criterion indexed by $k \in L$ is bounded. Since $d_{i} \leq 0$ for all $i \in M$, then for each $k \in L$

$$
d_{k} \geq \sum_{i \in M} a_{k i} d_{i} \leq 0
$$

If $d_{k} \geq 0$ for some $k \in L$ representing decay or no change in criterion $k$, then equation ( 8) holds. However, if $d_{k}<0$ for some $k \in L$ representing improvement in criterion $k$, then $\mathbf{d} \in W$ if and only if

$$
0>d_{k} \geq \sum_{i \in M} a_{k i} d_{i} \text { for each } k \in L \text { such that } d_{k}<0
$$

Inequality (9) shows that the total amount of improvement in criterion $k$ is bounded from below by the value of the expression $\sum_{i \in M} a_{k i} d_{i}$ calculated using the allowable tradeoff values and the amount of improvement in the relatively more important criteria in $M$.

Next we derive the algebraic cone representations of $P_{2}$. After all allowable tradeoff values are assigned by the DM, we define the following matrix.

Definition 3.5. Let $A_{2}$ be an $m \times m$ matrix with elements $\left(A_{2}\right)_{j}^{i}$ in row $i$ and column $j$ that are defined as follows:

$$
\begin{aligned}
& \left(A_{2}\right)_{i}^{i}=1 \quad \text { for all } i \in\{1,2, \ldots, m\} \\
& \left(A_{2}\right)_{j}^{i}=a_{i j} \text { for all } i \in L \text { and } j \in M \\
& \left(A_{2}\right)_{j}^{i}=0 \quad \text { otherwise }
\end{aligned}
$$

Using matrix $A_{2}$, define the related polyhedral cone.

$$
C\left(A_{2}\right):=\left\{\mathbf{d} \in \mathbb{R}^{m} \mid A_{2} \mathbf{d} \geqq \mathbf{0}\right\}
$$

Theorem 3.2. Let $D_{2}$ and $C\left(A_{2}\right)$ be defined as in (7) and (10), respectively. Then $D_{2}=C\left(A_{2}\right)$.

Due to its defining properties, the preference cone $C\left(A_{2}\right)$ is always convex and pointed.

The structure of $A_{2}$ depends on the definition of the sets of criterion indices $L$ and $M$. Suppose that for an MOP with four criteria, $L=\{1,2\}$ and $M=\{3,4\}$. Then $A_{2}$ has the following form:

$$
A_{2}=\left[\begin{array}{cccc}
1 & 0 & a_{13} & a_{14} \\
0 & 1 & a_{23} & a_{24} \\
0 & 0 & 1 & 0 \\
0 & 0 & 0 & 1
\end{array}\right]
$$


Note that the first two inequalities in the linear system $A_{2} \mathbf{d} \geqq \mathbf{0}$, corresponding to the first and second rows of matrix $A_{2}$, enforce that the first and second objectives may decay, remain unchanged, or improve according to (5). Note also that the last two inequalities in this system, corresponding to the third and fourth rows of matrix $A_{2}$, enforce that the third and fourth objectives may only improve or remain unchanged according to the assignment $M=\{3,4\}$. If $a_{i j}=0$ for any $i \in L$ and $j \in M$, then inequality $i$, corresponding to the $i$ th row of $A_{2}$, enforces that objective $i$ is not allowed to decay at all when objective $j$ improves and thus the cone will contain no directions $\mathbf{d}$ from the orthant where $d_{i}>0$ and $d_{j}<0$. Of course, if $a_{i j}=0$ for all $i \in L$ and $j \in M$, then none of the objectives in the problem are allowed to decay yielding the classical Pareto preference.

For a detailed development and complete derivations of these models, we refer the reader to Hunt (2004). For applications of these models in engineering design we refer the reader to Hunt and Wiecek (2003), Hunt et al. (2004, 2007), Wiecek (2007), and Blouin et al. (2007).

For illustration, Figure 1 depicts the set of outcomes given as $Y=\{y \in R$ : $\left.y_{1}^{2}+y_{2}^{2} \leq 1\right\}$ with three different domination cones in the two-dimensional outcome space, the Pareto cone $C_{1}=D_{\text {Par }}$, the polyhedral cone $C_{2}=D(A)$ for $A=\left[\begin{array}{ll}1 & 0 \\ 1 & 1\end{array}\right]$, and the $p$ th-order cone $C_{3}=C\left(\Gamma^{3}\right)=C_{p}^{m}$ that is defined in Section 5 .
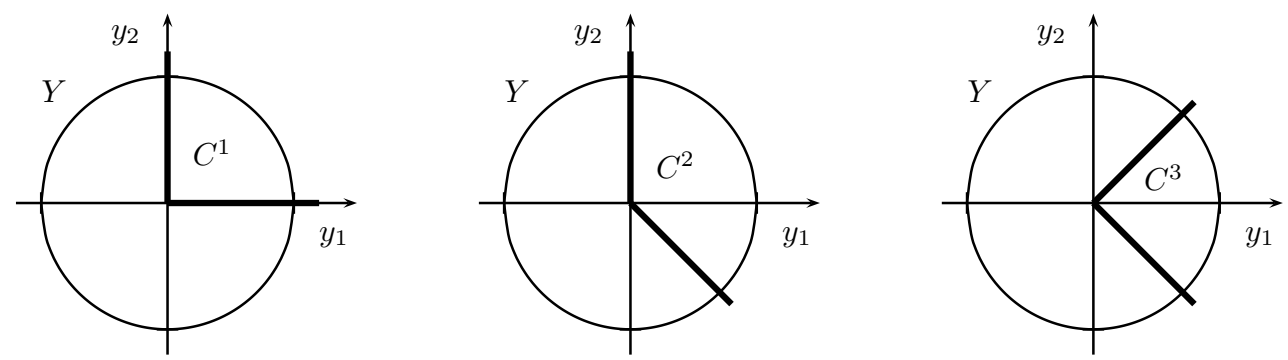

Fig. 1. The unit disk as outcome set $Y$ and the two-dimensional Pareto (left), polyhedral (center), and $p$ th-order cone (right) cone

\section{APPROXIMATE NONDOMINANCE}

In this section we use polyhedral cones to model approximate nondominance. In MOP, the notion of approximate nondominance was introduced by Kutateladze (1979), and later independently by Loridan (1984) and White (1986). Traditionally, approximate nondominance has been tolerable rather than desirable in MOP because it has been used in the context of modeling limitations or computational inaccuracies rather than for enhancing decision making. Engau (2007) and Engau and Wiecek (2007) explain 
reasons for enlarging the set of nondominated solutions with approximate nondominated solutions for decision making purposes. Such relaxation is useful when dealing with a collection of multiobjective programs for which a common preferred solution does not exist but the relaxation helps to find such a solution.

Definition 4.1. Let $\varepsilon \in D$. An element $\mathbf{y} \in Y$ is called an $\varepsilon$-nondominated element of the set $Y$ with respect to the domination cone $D$ if there do not exist an element $\mathbf{y}^{1} \in Y$ and a direction $\mathbf{d} \in D^{\circ}$ such that $\mathbf{y}=\mathbf{y}^{1}+\varepsilon+\mathbf{d}$, or equivalently, $Y \cap(\mathbf{y}-\varepsilon-$ $\left.D^{o}\right)=\emptyset$. The set of all $\varepsilon$-nondominated elements of $Y$ with respect to $D$ is denoted by $N(Y, D, \varepsilon)$. The set of weakly $\varepsilon$-nondominated elements of $Y$ with respect to $D$ is defined as $N_{w}(Y, D, \varepsilon):=N(Y$, int $D, \varepsilon)$.

Engau and Wiecek (2007) show that translated cones can be used to model the domination and preference cones of the $\varepsilon$-nondominance preference. Define first the translated cone according to Luenberger (1969).

Definition 4.2. Let $C \subset \mathbb{R}^{m}$ be a cone and $\varepsilon \in \mathbb{R}^{m}$ be a vector. Then the set $C_{\varepsilon}=C+\varepsilon$ is called a translated cone with the translation vector $\varepsilon$.

Let $D$ be a domination cone. The domination cone $D_{\varepsilon}$ of the $\varepsilon$-nondominance preference is defined as:

$$
D_{\varepsilon}:=D+\varepsilon
$$

and the preference cone $P_{\varepsilon}$ of the $\varepsilon$-nondominance preference is given as:

$$
P_{\varepsilon}=-D_{\varepsilon}
$$

Theorem 4.1. Let $D \subset \mathbb{R}^{m}$ be a convex pointed and $\varepsilon \in D$. Then

$$
N(Y, D, \varepsilon)=N\left(Y, D_{\varepsilon}\right)
$$

Engau (2007) and Engau and Wiecek (2007) extend the result of Theorem 2.1 for $\varepsilon$-nondominated elements of $Y$.

Theorem 4.2. Let $D \subset \mathbb{R}^{m}$ be a polyhedral cone given as $D=\left\{\mathbf{d} \in \mathbb{R}^{m} \mid A \mathbf{d} \geqq \mathbf{0}\right\}$, where $A$ is an $l \times m$ matrix, and let $\varepsilon \in D^{\circ}$ be a vector. Then

$$
A[N(Y, D, \varepsilon)] \subseteq N\left(A[Y], \mathbb{R}_{\geqq s}^{l}\right)
$$

where $\mathbb{R}_{\geqq s}^{l}:=\mathbb{R}_{\geqq}^{l}+\mathbf{s}=\left\{\mathbf{d} \in \mathbb{R}^{l}: \mathbf{d} \geqq \mathbf{s}\right\}$ and $\mathbf{s}=A \varepsilon$. If $D$ is pointed, then

$$
A[N(Y, D, \varepsilon)]=N\left(A[Y], \mathbb{R}_{\geqq s}^{l}\right)
$$

In any case,

$$
A\left[N_{w}(Y, D, \varepsilon)\right]=N_{w}\left(A[Y], \mathbb{R}_{\geqq s}^{l}\right)
$$


According to this result, the set of $\varepsilon$-nondominated elements of $Y$ with respect to a polyhedral pointed domination cone $D$ is equal to the set of nondominated elements of the set $A[Y]$ with respect to the translated Pareto domination cone with the translation vector $s=A \varepsilon$, where $A$ is the matrix of the polyhedral cone. In view of this result it is now possible to generate various types of $\varepsilon$-nondominated elements of $Y$ depending upon the structure of matrix $A$ describing the polyhedral cone. It is therefore possible to construct this matrix using the models of relative importance of criteria and in this way control the type of the $\varepsilon$-nondominated set for decision making purposes. The nondominated set may be enlarged due to allowing $\varepsilon$-nondominated elements or modified due to recognizing relative importance modeled by matrix $A$.

For applications of this preference in engineering design and portfolio optimization, the reader is referred to Engau (2007), Engau and Wiecek (2007) and Wiecek (2007).

\section{BEYOND POLYHEDRAL CONES}

In an effort to generalize polyhedral cones, we now make use of positively homogenous functions.

A function $\Gamma: \mathbb{R}^{m} \rightarrow \mathbb{R}^{r}$ is said to be positively homogeneous if $\Gamma(\lambda \mathbf{d})=\lambda \Gamma(\mathbf{d})$ whenever $\lambda>0$. If, in addition, $\Gamma\left(\mathbf{d}^{1}+\mathbf{d}^{2}\right) \leq \Gamma\left(\mathbf{d}^{1}\right)+\Gamma\left(\mathbf{d}^{2}\right)$ for all $\mathbf{d}^{1}, \mathbf{d}^{2} \in \mathbb{R}^{m}$, then $\Gamma$ is said to be sublinear. If, instead, $\Gamma\left(\mathbf{d}^{1}+\mathbf{d}^{2}\right) \geq \Gamma\left(\mathbf{d}^{1}\right)+\Gamma\left(\mathbf{d}^{2}\right)$ for all $\mathbf{d}^{1}, \mathbf{d}^{2} \in \mathbb{R}^{m}$, then $\Gamma$ is said to be superlinear. Finally, if, for all $\mathbf{d}^{1}, \mathbf{d}^{2} \in \mathbb{R}^{m}, \Gamma\left(\mathbf{d}^{1}\right)=\Gamma\left(\mathbf{d}^{2}\right)$ if and only if $\mathbf{d}^{1}=\mathbf{d}^{2}$, then $\Gamma$ said to be injective.

Definition 5.1. Let $\Gamma: \mathbb{R}^{m} \rightarrow \mathbb{R}^{r}$ be a positively homogeneous function. The nonpolyhedral cone $C(\Gamma) \subset \mathbb{R}^{m}$ induced by $\Gamma$ is defined by

$$
C(\Gamma):=\left\{\mathbf{d} \in \mathbb{R}^{m}: \Gamma(\mathbf{d}) \geqq \mathbf{0}\right\}
$$

In particular, the two functions $\Gamma^{1}(\mathbf{d})=\mathbf{d}$ and $\Gamma^{2}(\mathbf{d})=A \mathbf{d}$ induce the Pareto domination cone $C\left(\Gamma^{1}\right)=\mathbb{R}_{\geqq}^{m}$ and the polyhedral cone $C\left(\Gamma^{2}\right)=C(A)$, respectively.

Consider also the function $\Gamma^{3}(\mathbf{d})=d_{1}-\left\|\mathbf{d}_{-1}\right\|_{p}$. Given $p \geq 1$, this function induces the $p$-th order cone $C_{p}^{m} \subset \mathbb{R}^{m}$ defined by

$$
C\left(\Gamma^{3}\right)=C_{p}^{m}:=\left\{\mathbf{d}=\left(d_{1}, \mathbf{d}_{-1}\right) \in \mathbb{R}^{1+(m-1)}: d_{1} \geqq\left\|\mathbf{y}_{-1}\right\|_{p}\right\}
$$

For $p=2$, the second order cones $C_{2}^{m}$ are also called Lorentz or ice cream cones (see also Figure 1).

In Engau (2007) it is proven that $C(\Gamma)$ is a cone. If $\Gamma$ is superlinear then $C(\Gamma)$ is convex. If, in addition, the condition $\Gamma(\mathbf{y})=0$ if and only if $\mathbf{y}=0$ holds, then $C(\Gamma)$ is pointed. If the function $\Gamma$ is injective, then this condition is always satisfied.

Given a positively homogeneous function $\Gamma$, the domination cone $D(\Gamma)$ is defined as

$$
D(\Gamma):=\left\{\mathbf{d} \in \mathbb{R}^{m}: \Gamma(\mathbf{d}) \geqq \mathbf{0}\right\} .
$$

and the preference cone is given by

$$
P(\Gamma)=-D(\Gamma)
$$


The result of relating the set of nondominated solutions with respect to polyhedral cones or translated polyhedral cones to the nondominated set with respect to the Pareto cone also holds for nonpolyhedral cones induced by a positively homogeneous function.

Theorem 5.1. Let $\Gamma: \mathbb{R}^{m} \rightarrow \mathbb{R}^{r}$ be a positively homogeneous function and $D(\Gamma) \subset$ $\mathbb{R}^{m}$ be the induced domination cone. If $\Gamma$ is sublinear, then

$$
\Gamma[N(Y, D(\Gamma))] \subseteq N\left(\Gamma[Y], \mathbb{R}_{\geqq}^{r}\right)
$$

If $\Gamma$ is superlinear and injective, then

$$
\Gamma[N(Y, D(\Gamma))] \supseteq N\left(\Gamma[Y], \mathbb{R}_{\geqq}^{r}\right)
$$

If $\Gamma$ is linear and injective, then

$$
\Gamma[N(Y, D(\Gamma))]=N\left(\Gamma[Y], \mathbb{R}_{\geqq}^{r}\right)
$$

Since this result makes preferences modeled with nonpolyhedral cones available for MOP, it is of interest to explore the meaning of these preferences in contrast to those modeled with polyhedral cones. In general, polyhedral cones can be viewed as piecewise linear approximation of nonpolyhedral cones. Since polyhedral cones model relative importance of criteria in a piecewise linear fashion, nonpolyhedral cones might model continuous change of this importance. This, however, remains for now an open research question.

\section{EQUITABILITY WITH VARIABLE CONES}

It is surprising but even further generalization of cones into a bigger family than nonpolyhedral cones was already introduced by $\mathrm{Yu}$ (1974). All the cones used for preference modeling so far in this paper are constant cones in the sense that the domination (preference) cone is the same at every point $\mathbf{y} \in \mathbb{R}^{m}$. However, Yu (1974) also proposes to use variable cones to define nondominated outcomes with respect to structures of domination.

In general, let $C(\mathbf{y}) \subset \mathbb{R}^{m}$ denote a cone at $\mathbf{y} \in \mathbb{R}^{m}$. In general, $C\left(\mathbf{y}^{1}\right) \neq C\left(\mathbf{y}^{2}\right)$ for $\mathbf{y}^{1}, \mathbf{y}^{2} \in \mathbb{R}^{m}, \mathbf{y}^{1} \neq \mathbf{y}^{2}$.

Definition 6.1. Let the family $\mathcal{D}=\{D(\mathbf{y}), \mathbf{y} \in Y\}$, where $D(\mathbf{y})$ is a domination convex cone in $\mathbb{R}^{m}$ for each $\mathbf{y} \in \mathbb{R}^{m}$, be called the structure of domination. An element $\mathbf{y} \in Y$ is called a nondominated element of the set $Y$ with respect to the structure of domination $\mathcal{D}$ if there does not exist an element $\mathbf{y}^{1} \in Y$ such that $\mathbf{y} \in \mathbf{y}^{1}+D\left(\mathbf{y}^{1}\right) \backslash\{\mathbf{0}\}$. The set of all nondominated elements of $Y$ with respect to $\mathcal{D}$ is denoted by $N(Y, \mathcal{D})$.

If $D(\mathbf{y})=D$ for each $\mathbf{y} \in \mathbb{R}^{m}$, then the concept of nondominance in Definition 6.1 reduces to the nondominance with respect to constant cones in Definition 2.2.

Let $P(\mathbf{y})$ in $\mathbb{R}^{m}$ denote the preference convex cone for $\mathbf{y} \in Y$. The property that $P=-D$ available for constant cones does not hold true for the variable cones defined above. In general, $P(\mathbf{y}) \neq-D(\mathbf{y})$. 
This very general concept of nondominance allows for using a different domination cone at each point of the space. In a practical context, one may say that preferences depend upon the current values of objective functions or the so-called "decisional wealth" (see Karaskal and Michalowski (2003)). For example, when a criterion performs poorly or unsatisfactorily, its very small improvement may be very desirable even that it may cause other "rich" criteria to decay. However, when a criterion performs very well, its small improvement would be unimportant and certainly undesirable if it caused other "poor" criteria to further decay.

A well researched example of a preference based on variable cones is the preference of equitability introduced to MOP by Kostreva and Ogryczak (1999) and further examined by Kostreva et al. (2004). It strengthens the concept of Pareto nondominance by additionally requiring that the objective functions be comparable (measured on a common scale) and anonymous (impartial), and satisfy the Pigou-Dalton principle of transfers. The former makes the distribution of outcomes among the criteria more important than the assignment of outcomes to specific criteria. The latter means that any outcome with two unequal components can be improved by transferring a ceratin amount from the larger to the smaller component to reduce the difference (inequity) between the corresponding criteria. Both these additional requirements model equitability among the criteria.

Baatar and Wiecek (2006) developed the structure of domination for this preference. Let $I_{k}, k=1, \ldots, m$ !, denote the matrices obtained by permuting columns of the $m \times m$ identity matrix. Let $E$ denote the $m \times m$ lower triangular matrix of the form

$$
E=\left[\begin{array}{ccccc}
1 & 0 & 0 & \ldots & 0 \\
1 & 1 & 0 & \ldots & 0 \\
\ldots & \ldots & \ldots & \ldots & \ldots \\
1 & 1 & 1 & \ldots & 1
\end{array}\right]
$$

Due to variability of this preference, the space $\mathbb{R}^{m}$ has to be partitioned into sectors.

Definition 6.2. The set $S_{i} \subset \mathbb{R}^{m}, i=1, \ldots, m$ !, defined as

$$
S_{i}:=\left\{\mathbf{y} \in \mathbb{R}^{m}:\left(I_{i} \mathbf{y}\right)_{1} \geq\left(I_{i} \mathbf{y}\right)_{2} \geq \cdots \geq\left(I_{i} \mathbf{y}\right)_{m}\right\}
$$

where $\left(I_{i} \mathbf{y}\right)_{j}$ is the $j$-th component of the vector $I_{i} \mathbf{y}$, is called a sector $i$.

We also define an auxiliary polyhedral cone by means of the matrix $E$ and the permutation matrix $I_{k}, k=1, \ldots, m$ !.

Definition 6.3. The polyhedral cone $D_{k} \subset \mathbb{R}^{m}, k=1, \ldots, m$ !, of the form

$$
D_{k}:=\left\{\mathbf{d} \in \mathbb{R}^{m}: E I_{k} \mathbf{d} \geqq \mathbf{0}\right\}
$$

is called a permutation cone.

The permutation cone $D_{k}$ is convex and pointed.

In Baatar and Wiecek (2006) it shown that the domination and preference cones of the equitabiliy preference are variable and depend upon the location of the outcome $y$ in a sector $S_{k}$. 
Theorem 6.1. Let $\mathbf{y} \in S_{k}$. The set $D(\mathbf{y}) \subset \mathbb{R}^{m}$

$$
D(\mathbf{y})=\bigcup_{p=1}^{m !} I_{p}^{T} I_{k}\left(\mathbf{y}+D_{k}\right)
$$

is the domination cone of the equitability preference at $\mathbf{y} \in S_{k}$. The set $P(\mathbf{y}) \subset \mathbb{R}^{m}$

$$
P(\mathbf{y})=\bigcap_{p=1}^{m !} I_{p}^{T} I_{k}\left(\mathbf{y}-D_{k}\right)
$$

is the preference cone of the equitability preference at $\mathbf{y} \in S_{k}$.

Obviously, $P(\mathbf{y}) \neq-D(\mathbf{y})$. Additionally, $P(\mathbf{y})$ is a convex set while $D(\mathbf{y})$ is not.

In order to find the nondominated outcomes in $\mathrm{Y}$ with respect to $D(\mathbf{y})$, one may extend the last part of Theorem 2 in Ehrgott and Wiecek (2005) to multiple cones $C_{k} \subset \mathbb{R}^{m}, k=1, \ldots, K$ :

$$
N\left(Y, \bigcup_{k=1}^{K} C_{k}\right)=\bigcap_{k=1}^{K} N\left(Y, C_{k}\right)
$$

Let $\mathbf{y} \in S_{k}$, and $D_{k, y}=\mathbf{y}+D_{k}$ be a translated cone with the translation vector $\mathbf{y}$.

$$
N(Y, D(\mathbf{y}))=N\left(Y, \bigcup_{p=1}^{m !} I_{p}^{T} I_{k}\left(\mathbf{y}+D_{k}\right)\right)=\bigcap_{p=1}^{m !} N\left(Y, I_{p}^{T} I_{k} D_{k, y}\right)
$$

In effect, the problem of finding equitably nondominated points with respect to $D(\mathbf{y})$ can be decomposed into $m$ ! problems of finding nondominated points in $\mathrm{Y}$ with respect to a (constant) translated polyhedral cone. While other researchers work on finding methods for generating equitable solutions (e.g., Singh (2007)), the knowledge of the domination cone for this preference may open another way to accomplish this goal.

For applications of the equitability preference in location, portfolio analysis, telecommunication and others the reader is referred to Ogryczak (1997, 2000), Ogryczak et al. (2008) and Singh (2007).

\section{CONCLUSION}

This paper presents an overview of the most recent advances in cone-based preference modeling in MOP. The classical Pareto preference is considered as one type in the family of preferences that are all modeled with cones. The family also includes the relative importance preference modeled with polyhedral cones, the approximate nondominance modeled with translated cones, preferences modeled with nonpolyhedral cones, and the preference of equitability modeled with variable cones. For each preference, the domination and preference cones as well as the results on finding the related 
nondominated set are given. Furthermore, the significance of using these preferences in decision making is discussed.

The overview makes use of many results spread out in the operations research and engineering literature in various articles, reports, and theses. The reader interested either in theoretical details or applications is referred to those sources for proofs, complete derivations, and examples. This overview however presents all those results from a brief but unified perspective and with common notation.

The author hopes that the paper attests to close interplay between theory and practice of decision making and will stimulate further interest and investigation in this inspiring area of research.

This research was partially supported by the National Science Foundation, grant number CMMI 0621055.

\section{REFERENCES}

1. D. Baatar, M.M. Wiecek, Advancing equitability in multiobjective programming, Computers and Mathematics with Applications, 52 (2006) 1-2, 225-234.

2. K. Bergstresser, A. Charnes, P.L. Yu, Generalization of domination structures and nondominated solutions in multicriteria decision making, Journal of Optimization Theory and Applications, 18 (1976) 1, 3-13.

3. V.P. Berman, G.E. Naumov, Preference relation and interval value tradeoffs in criterion space, Automation and Remote Control 50 (1989) 3, 398-410.

4. V.Y. Blouin, B.J. Hunt, M.M. Wiecek, Relative importance of criteria in configuration design of vehicles, Department of Mathematical Sciences, Clemson University, Technical Report 2007.

5. A. Cambini, D.T. Luc, L. Martein, Order-preserving transformations and applications, Journal of Optimization Theory and Applications, 118 (2003) 2, 275-293.

6. G.Y. Chen, X. Huang, X. Yang, Vector Optimization. Set-valued and Variational Analysis. Springer, Berlin (2005).

7. G.Y. Chen, X. Q. Yang, Characterization of variable domination structures via nonlinear scalarization, Journal of Optimization Theory and Applications, 112 (2002) 1, 97-110.

8. K.L. Chew, Domination structures in abstract spaces, Southeast Asian Bulletin of Mathematics, Proceedings of the First Franco-Southeast Asian Mathematical Conference 2 (1979), 190-204.

9. J. Doležal, Hierarchical solution concept for static and multistage decison problems with two objectives, Kybernetika 12 (1976) 5, 363-385.

10. M. Ehrgott, M.M. Wiecek, Multiobjective Programming. In: Multiple Criteria Decision Analysis: State of the Art Surveys (eds. J. Figueira, S. Greco, and M. Ehrgott). Springer, New York, (2005), 667-722.

11. A. Engau, Domination and Decomposition in Multiobjective Programming. PhD Thesis, Clemson University (2007). 
12. A. Engau, M.M. Wiecek, Cone characterizations of approximate solutions in real vector optimization, Journal of Optimization Theory and Applications 134 (2007) 3 (in press).

13. A. Engau, M. M. Wiecek, Generating $\epsilon$-efficient solutions in multiobjective programming, European Journal of Operational Research 177 (2007) 3, 1566-1579.

14. A. Engau, M.M. Wiecek, Exact generation of epsilon-efficient solutions in multiple objective programming, OR Spectrum 29 (2007) 2, 335-350.

15. A. Engau, M.M. Wiecek, 2D decision making for multi-criteria design optimization, Structural and Multidisciplinary Optimization 2007 (in press).

16. J. Figueira, S. Greco, M. Ehrgott, Multiple Criteria Decision Analysis: State of the Art Surveys, Springer, New York (2005).

17. G.B. Hazen, T.L. Morin, Optimality conditions in nonconical multiple-objective programmin, Journal of Optimization Theory and Applications, 40 (1983) 1, 25-60.

18. B.J. Hunt, Multiobjective Progarmming with Convex Cones: Methodology and Applications. PhD Thesis, Clemson University (2004).

19. B.J. Hunt, V.Y. Blouin, M.M. Wiecek, Modeling relative importance of design criteria with a modified Pareto preference, Journal of Mechanical Design, 2007 (in press).

20. B.J. Hunt, M.M. Wiecek, Cones to aid decision making in multicriteria programming, In: Multiobjective Programming and Goal Programming (eds. T. Tanino, T. Tanaka, and M. Inuiguchi). Springer, Berlin, (2003), 153-158.

21. B.J. Hunt, M.M. Wiecek, G. Fadel, Matrices as preference modeling tools in bi-criteria engineering design, 10th AIAA/ISSMO Multidisciplinary Analysis and Optimization Conference, Albany, August-Sepetember AIAA (2004), 2004-4320.

22. E.K. Karaskal, W. Michalowski, Incorporating wealth information into a multiple criteria decision making model, European Journal of Operational Research 150 (2003), 204-219.

23. M.M. Kostreva, W. Ogryczak, Linear optimization with multiple equitable criteria, RAIRO Operations Research, 33 (1999) 3, 275-297.

24. M.M. Kostreva, W. Ogryczak, A. Wierzbicki, Equitable aggregations in multiple criteria analysis, European Journal of Operational Research, 158 (2004) 2, 362-377.

25. S.S. Kutateladze, Convex E-programming, Soviet Mathematics. Doklady 20 (1979) 2, 391-393.

26. S.A.Y. Lin, A comparison of Pareto optimality and domination structure, Metroeconimica, 28 (1976) 1-3, 62-74.

27. P. Loridan, $\varepsilon$-Solutions in vector minimization problems, Journal of Optimization Theory and Applications, 43 (1984) 2, 265-276.

28. D.G. Luenberger, Optimization by Vector Space Methods, John Wiley and Sons, New York (1969).

29. O.R. Men'shikova, V.V. Podinovskii, Constructing the preference relation and the core in multicriterion problems with inhomogenous criteria ordered by importance, U.S.S.R. Computational Matehmatics and Mathematical Physics 28 (1988) 3, 15-22. 
30. V.D. Noghin, Relative importance of criteria: A quantitative approach, Journal of Multi-criteria Decision Analysis, 6 (1997), 355-363.

31. V.D. Noghin, I.V. Tolstykh, Using quantitative information on the relative importance of criteria for decision making, Computational Matehmatics and Mathematical Physics 40 (2000) 11, 1529-1536.

32. W. Ogryczak, Multiple criteria linear programming model for portfolio selection, Annals of Operations Research, 97 (1997), 143-162.

33. W. Ogryczak. Inequality measures and equitable approaches to location problems, European Journal of Operational Research, 122 (2000) 2, 374-391.

34. W. Ogryczak, A. Wierzbicki, M. Milewski, A multi-criteria approach to fair and efficient bandwidth allocation, Omega 36 (2008) 3, 451-463.

35. V.V. Podinovskii, Two-criterial problems with unequally rated criteria, Engineering Cybernetics 5 (1977), 44-59.

36. V.V. Podinovskii, Importance coefficients of criteria in decision making problems: serial or ordinal importance coefficients, Automation and Remote Control 39 (1978) 10, $1514-1524$.

37. V.V. Podinovskii, Criteria imporance theory, Mathematical Social Sciences 27 (1994), $237-252$.

38. V.V. Podinovskii, Quantitative importance of criteria, Automation and Remote Control 61 (2000) 5, 817-828.

39. B. Roy, V. Mousseau, A theoretical framework for analyzing the notion of relative importance of criteria, Journal of Multi-criteria Decision Analysis, 5 (1996), 145-159.

40. Y. Sawaragi, H. Nakayama, T. Tanino, Theory of Multiobjective Optimization. Academic Press, Orlando, 1985.

41. V.K. Singh, Equitable Efficiency in Multiple Criteria Optimization. PhD Thesis, Clemson University 2007.

42. E. Takeda, T. Nishida, Multiple criteria decision problems with fuzzy domination structures, Fuzzy Sets and Systems, 3 (1980) 123-136.

43. Q. Wei, H. Yan, J. Ma, Z. Fan, A compromise weight for multicriteria group decsion making with individual preference, Journal of Operational Research Society 51 (2000), 625-634.

44. P. Weidner, Problems in scalarizing multicriteria approaches, In Multiple Criteria Decision Making in the New Millennium (eds. S. Zionts and and M. Köksalan). Springer, Berlin, (2001), 199-209.

45. P. Weidner, Tradeoff directions and dominance sets, In: Multi-Objective Programming and Goal Programming (eds. T. Tanino, T. Tanaka, and M. Inuiguchi). Springer, Berlin, (2003), 275-280.

46. R.E. Wendell, D.N. Lee, Efficiency in multiple objective optimization problems, Mathematical Programming, 12 (1977) 3, 406-414. 
47. D.J. White, Epsilon efficiency, Journal of Optimization Theory and Applications 49 (1986) 2, 319-337.

48. M.M. Wiecek, Multi-scenario multi-objective optimization for engineering design, In: Advances in Computational Optimization and its Applications (eds. K. Deb, P. Chakroborty, N.G.R. Iyengar, and S.K. Gupta). Universities Press, India, (2007), $170-174$

49. H.C. Wu, A solution concept for fuzzy multiobjective programming problems based on convex cones, Journal of Optimization Theory and Applications 121 (2004) 2, 397-417.

50. M.Q. Ying, The set of cone-extreme points and the grouping hierarchy problem, Journal of Systems Science and Mathematical Sciences 3 (1983) 2, 125-138.

51. P.L. Yu, Cone convexity, cone extreme points and nondominated solutions in decision problems with multiobjectives. Journal of Optimization Theory and Applications, 14 (1974), 319-377.

52. P.L. Yu, Multiple-Criteria Decision Making: Concepts, Techniques, and Extensions Plenum Press, New York 1985. 